\title{
ƯNG DỤNG ĐIỀU KHIỂN MỜ TRONG BÀI TOÁN ROBOT BÁM QỮ ĐẠO
}

\author{
Mạc Thị Thoa*, Vũ Văn Thích, Trần Đức Trung \\ Viện co khí, Truoòng Đại học Bách khoa Hà Nội, Số 1, Đại Cồ Việt, Hà Nội \\ *Email: thoa.macthi@hust.edu.vn
}

Đến Tòa soạn: 1/7/2014; Chấp nhận đăng: 14/1/2015

\begin{abstract}
TÓM TĂT
Điều khiển mờ hay còn gọi là điều khiển thông minh, mô phỏng trên phương thức xử lí thông tin và điều khiển của con người, khởi đầu cho sự ứng dụng của trí tuệ nhân tạo trong lĩnh vực điều khiển. Nghiên cứu trình bày phương pháp điều khiển robot tự hành trên cơ sở logic mờ. Trước tiên, mô hình động học robot được xây dựng, sau đó được mô hình hóa để mô phỏng quá trình bám quỹ đạo cho trước trong không gian làm việc của robot. Kết quả mô phỏng cho thấy tính đúng đắn, hiệu quả của giải thuật điều khiển.
\end{abstract}

Tù khóa: thiết kế quỹ đạo, điều khiển mờ, robot tự hành, bám quỹ đạo, điều khiển động học.

\section{GIỚI THIỆU}

Thiết kế quỹ đạo và thiết lập bộ điều khiển để bám theo quỹ đạo này là nhiệm vụ rất quan trọng của robot di động. Trong các nghiên cứu gần đây thường giải quyết các vấn đề sau: điều khiển robot bám vạch, bám tường, bám quỹ đạo, tránh vật cản, đi tới điểm đích ....

Robot di động được nghiên cứu là mô hình robot 3 bánh gồm 2 biến điều khiển (vận tốc bánh trái và vận tốc bảnh phải), vị trí của robot được xác định thông qua ba biến trạng thái (x,y, $\theta)$. Các phương pháp điều khiền ngày càng thông minh hơn bằng cách sử dụng mạng neron $[1$, 2] và Logic mờ $[3,4]$. Do tính mềm dẻo của nó, các bộ điều khiển mờ ngày càng được ứng dụng rộng rãi và có kết quả đáng ghi nhận [5 - 10].

Bài báo trình bày cách tiếp cận mới trong thiết kế bộ điều khiển mờ để robot bám theo các quỹ đạo tuyến tính, phi tuyến bất kỳ trong không gian làm việc của nó. Bố cục bài báo được sắp xếp như sau: Phần 2 là phương pháp nghiên cứu, trong phần này đưa ra mô hình động học robot, mô hình điều khiển; kết quả mô phỏng và thảo luận được trình bày ở phần 3 ; kết luận của nghiên cứu viết trong phần 4 , cuối cùng là danh mục các bài báo tham khảo.

\section{PHƯƠNG PHÁP NGHIÊN CÚU}

\subsection{Mô hình động học robot}


Trong nghiên cứu này sử dụng mô hình robot gồm 3 bánh, 2 bánh chủ động phía sau gắn 2 động cơ, 1 bánh lái phía trước có khả năng quay tự do trong đó mô hình bánh xe robot được lí tưởng hóa như Hình 1. Bánh xe quay quanh trục của nó (trục $Y$ ), chuyển động theo phương $X$ (trục $\mathrm{X}$ ). Khi chuyển động ở tốc độ thấp, có thể bỏ qua ảnh hưởng của sự trượt của bánh xe so với mặt đường.

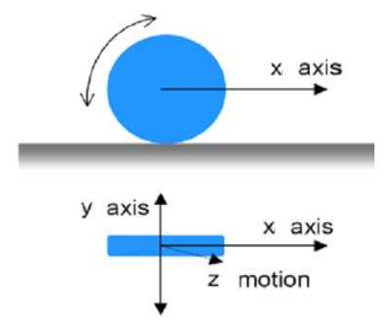

Hình 1. Mô hình bánh xe robot đã được lí tưởng hóa.

trong đó: $\mathrm{X}$ là phương chuyển động của xe;

Y vuông góc với phương chuyển động;

$\mathrm{Z}$ thẳng đứng, vuông góc $\mathrm{X}, \mathrm{Y}$.

Tọa độ của robot trong mặt phảng $2 \mathrm{D}-\mathrm{X}_{\mathrm{m}} \mathrm{GY}_{\mathrm{m}}$ tại một thời điểm bất kỳ được xác định trong hệ tọa độ cố định gắn với tâm robot và góc của đầu robot so với hệ tọa độ gốc tham chiếu $\mathrm{X}_{\mathrm{b}} \mathrm{GY}_{\mathrm{b}}$ (Hình 2). Vậy vị trí robot sẽ được xác định theo vectơ q (3x1).

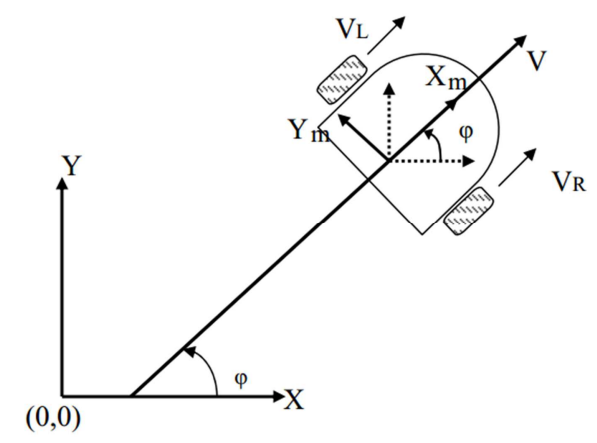

Hình 2. Mô hình Robot xe ba bánh.

$$
q=
$$

Ma trận quay biểu diễn hướng của tọa độ cố định so với hệ tọa độ động gắn với tâm robot là:

Động học đưa ra mô hình toán học của robot chỉ quan tâm đến quan hệ hình học thuần túy của hệ thổng mà bỏ qua tác động của lực đến chuyển động của robot. Mô hình đưa ra mối quan 
hệ giữa các biến điều khiển và đặc tính hệ thống. Giả sử vị trí ban đầu của robot là $(X, Y)$, và góc tạo bởi vecto vận tốc $\mathrm{V}$ với trục ngang là .

Vận tốc của bánh trái và bánh phải (vleft, vright) liên hệ với vận tốc góc như sau:

$$
\begin{aligned}
& v_{\text {right }}=r * \omega_{\text {right }} \\
& v_{\text {left }}=r * \omega_{\text {left }}
\end{aligned}
$$

trong đó: $\mathrm{r}$ là bán kính bánh xe, $\omega_{\text {right }}, \omega_{\text {left }}$ lần lượt là vận tốc góc của bánh trái và bánh phải. Giả thiết $\mathrm{V}_{\mathrm{r}}, \omega_{\mathrm{r}}$ là vận tốc và vận tốc góc của robot, $\mathrm{d}$ là khoảng cách giữa hai bánh sau. Robot luôn quay quanh tâm quay tức thời là giao điểm của 2 trục bánh xe. Điểm này gọi là tâm quay tức thời (ICC - Instantaneous Centreof Curvature). Công thức tính như sau:

$$
\begin{gathered}
\mathrm{v}_{\mathrm{R}}=\left(\mathrm{v}_{\text {right }}+\mathrm{v}_{\text {left }}\right) / 2 \\
-- \\
- \\
- \\
=\omega_{\mathrm{R}}
\end{gathered}
$$$$
-
$$

Mô hình động học robot viết trong hệ tọa độ Đềcác như sau:

Thay phương trình (7), (8) vào (12), biến đổi đơn giản ta thu được:

Phương trình (11) chính là phương trình động học robot tự hành.

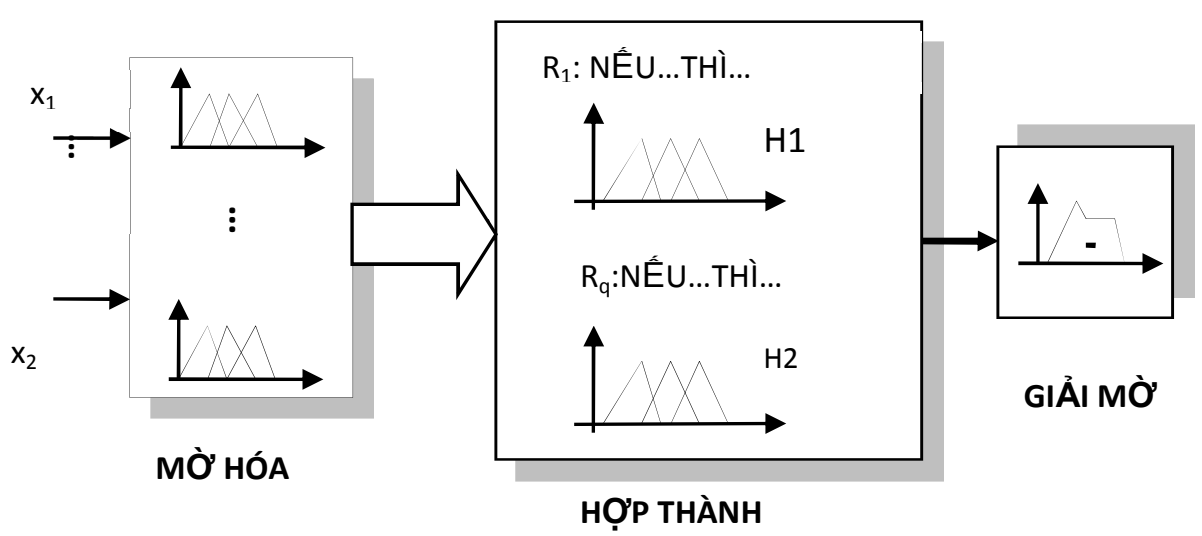

Hình 3. Bộ điều khiển mờ. 
Dựa trên mô hình động học của robot, các phương pháp điều khiển có thể áp dụng là: điều khiển PID, điều khiển trượt, điều khiển mờ, điều khiển dựa trên mạng neron .... Trong nghiên cứu này sử dụng bộ điều khiển mờ (Hình 3 ). Bộ điều khiển mờ dựa trên kinh nghiệm, dùng các luật ngôn ngữ IF- THEN để điều khiển đặc tính của robot. Ủu điểm của phương pháp này là tín hiệu điều khiển trơn.

Đầu vào của bộ mờ hóa là các biến sai số vị trí và góc của robot di động so với quỹ đạo thiết kế, sau bộ giải mờ là vận tốc góc của bánh trái và vận tốc bánh phải robot. Các biến đầu vào/ ra sử dụng hàm tam giác để giảm thời gian tính toán mà vẫn đạt được độ chính xác cần thiết. Các luật hợp thành là phần khó nhất trong quá trình thiết kế, dựa trên kinh nghiệm về điều khiển và mô phỏng robot, được trình bày cụ thể ở phần 2.2 .

\section{2 Ứng dụng bộ điều khiển mờ vào điều khiển robot bám theo quỹ đạo.}

Một cách tổng quát, quỹ đạo cho robot được thiết kế dưới dạng vecto $3 \mathrm{x} 1\left(\mathrm{x}_{\mathrm{p}}, \mathrm{y}_{\mathrm{p}}, \theta_{\mathrm{p}}\right)$. Đầu vào của bộ điều khiển mờ sẽ là giá trị khoảng cách (Distance) và góc lệch (Angle ). Đầu ra của bộ điều khiển mờ sẽ là vận tốc góc bánh phải và trái $\left(\mathrm{w}_{\mathrm{r}}, \mathrm{w}_{1}\right)$. Trong khi robot chuyển động các yếu tố như nhiễu, ma sát và các ngoại lực được bỏ qua.

Sơ đồ khối điều khiển được mô tả trong Hình 4.

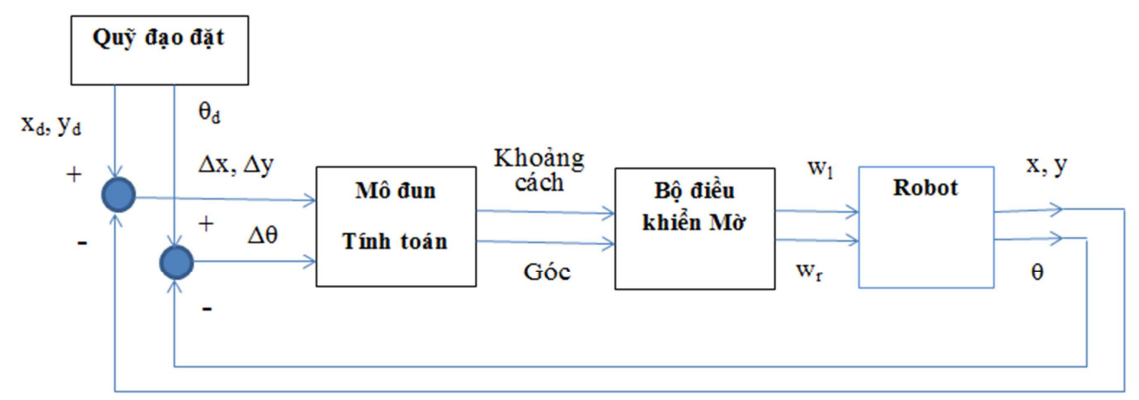

Hình 4. Sơ đồ điều khiển mờ robot bám theo quỹ đạo.

Trong mô hình này, quỹ đạo thiết kế cho ta vecto $\left(\mathrm{x}_{\mathrm{d}}, \mathrm{y}_{\mathrm{d}}, \theta_{\mathrm{d}}\right)$ - đây là quỹ đạo bám (reference) của robot. Mô đun tính tính toán so sánh giá trị tọa độ thực tế $(\mathrm{x}, \mathrm{y}, \theta)$ với giá trị đặt $(\Delta \mathrm{x}, \Delta \mathrm{y}, \Delta \theta)$ để tính chệnh lệch về khoảng và hướng (Distance và Angle) làm tín hiệu đầu vào cho bộ điều khiển mờ. Vecto sai số trong modun toán được xác định như sau:

Robot được mô hình hóa dựa trên phương trình (11) trong simulink - matlab như sau: 


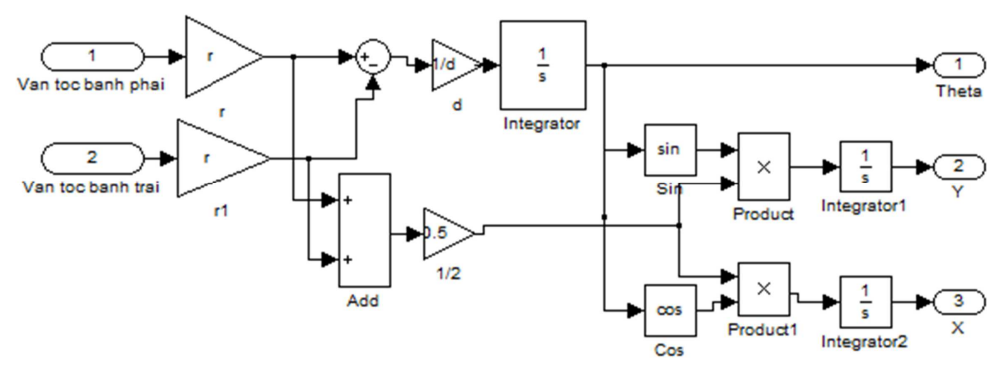

Hình 5. Mô hình động học robot trong Simulink.

Bộ điều khiển mờ được xây dựng như sau:

a. Xác đinh của tập mò̀

Miền giá trị vật lí cơ sở của các biến ngôn ngữ

- Tín hiệu đầu vào, sai lệch về khoảng cách: $0 \leq$ Distance $\leq 10(\mathrm{~cm})$

- Tín hiệu đầu vào, sai lệch về góc: - - i $\leq$ Angle $\leq$ pi (rad)

- Tín hiệu đầu ra, vận tốc góc bánh trái, phải: $0 \leq \omega_{\text {right }} \leq 6 \mathrm{rad} / \mathrm{s}$

b. Số lượng tập mờ (giá trị ngôn ngũ)

$0 \leq \omega_{\text {right }} \leq 6 \mathrm{rad} / \mathrm{s}$

Về nguyên tắc, số lượng tập mờ cho mỗi biến ngôn ngữ phải được lựa chọn hợp lí, nếu số lượng quá nhỏ thì không thực hiện được việc chi tiểt hóa các phương án xử lí. Ngược lại, số lượng biến ngôn ngữ lớn thì người lập trình phải đưa ra khá lớn các phương án suy luận, đồng thời tín hiệu truyền đến robot có thể bị trễ do mất nhiều thời gian tính toán. Trong bài toán đạt ra, tác giả chọn các biến ngôn ngữ như sau:

- Sai lệch về khoảng cách: Distance (Zero, Near, Medium, Far, Very far)

- Sai lệch về góc: Angle (Bignegative, Negative, Zero, Positive, Big positive)

- Vận tốc góc bánh trái, phải: $\omega_{\text {left }}, \omega_{\text {right }}$ (Veryslow, Slow, Medium, Fast, Veryfast)

c. Các hàm liên thuộc

Bảng 1. Thông số biến Distance.

\begin{tabular}{|c|c|c|}
\hline \multicolumn{3}{|c|}{ Thông số Distance(cm) } \\
\hline Tên gọi & Viết tắt & Miền giá trị \\
\hline Zero & Zero & {$\left[\begin{array}{lll}-2.5 & 0 & 2.5\end{array}\right]$} \\
\hline Near & $\mathrm{N}$ & {$\left[\begin{array}{lll}0 & 2.5 & 5\end{array}\right]$} \\
\hline Medium & $\mathrm{M}$ & {$\left[\begin{array}{lll}2.5 & 5 & 7.5\end{array}\right]$} \\
\hline Far & $\mathrm{F}$ & {$\left[\begin{array}{lll}5 & 7.5 & 10\end{array}\right]$} \\
\hline Very far & VF & {$\left[\begin{array}{llll}7.5 & 10 & 12.5\end{array}\right]$} \\
\hline
\end{tabular}




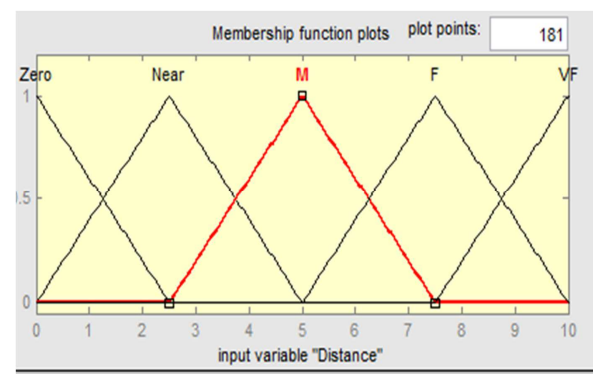

Hình 6. Miền xác định các hàm thành viên Distance.

Điều kiện là phải chọn các hàm liên thuộc có phần chồng lên nhau phủ kín miền giá trị vật lí để tránh trường hợp vẫn có tín hiệu đầu vào mà bộ điều khiển không đưa ra được một quyểt định điều khiển nào. Ở đây chọn mỗi biến ngôn ngữ gán với một hàm phụ thuộc dạng tam giác. Dựa vào các phân tích trên đây ta đưa vào phương trình mô phỏng như Bảng 1 . như sau:

Biến giá trị đầu vào Angle, có miền xác định -1 rad đến 1 rad, với các hàm được xác định

Bảng 2. Bảng thông số Angle.

\begin{tabular}{|c|c|c|}
\hline \multicolumn{3}{|c|}{ Thông số Angle(rad) } \\
\hline Tên gọi & Viết tắt & Miền giá trị \\
\hline Bignegative & $\mathrm{BN}$ & {$\left[\begin{array}{lll}-1.5 & -1 & -0.5\end{array}\right]$} \\
\hline Negative & $\mathrm{N}$ & {$\left[\begin{array}{lll}-1 & -0.5 & 0\end{array}\right]$} \\
\hline Zero & $\mathrm{Z}$ & {$\left[\begin{array}{lll}-0.5 & 0 & 0.5\end{array}\right]$} \\
\hline Positive & $\mathrm{P}$ & {$\left[\begin{array}{lll}0 & 0.5 & 1\end{array}\right]$} \\
\hline Bigpositive & $\mathrm{BP}$ & {$\left[\begin{array}{lll}0.5 & 1 & 1.5\end{array}\right]$} \\
\hline
\end{tabular}

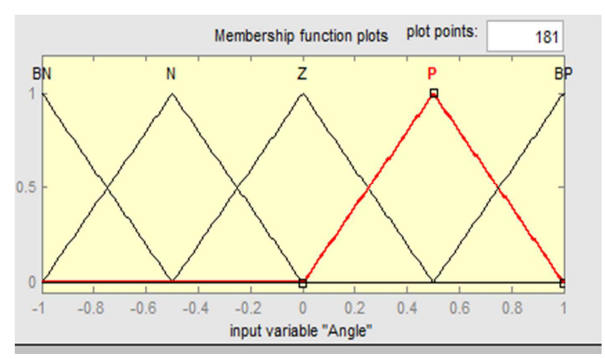

Hình 7. Miền xác định các hàm thành viên Angle.

Biến giá trị đầu ra gồm vận tốc bánh phải và bánh trái. Chúng có miền xác định là 0-6 $\mathrm{rad} / \mathrm{s}$. 
Bảng 3. Bảng thông số bánh phải và bánh trái.

\begin{tabular}{|c|c|c|}
\hline \multicolumn{3}{|c|}{ Thông số bánh phải, bánh trái(rad/s) } \\
\hline Tên gọi & Viết tắt & Miền giá trị \\
\hline Veryslow & VS & {$\left[\begin{array}{lll}-1.5 & 0 & 1.5\end{array}\right]$} \\
\hline Slow & $\mathrm{S}$ & {$\left[\begin{array}{lll}0 & 1.5 & 3\end{array}\right]$} \\
\hline Medium & $\mathrm{M}$ & {$\left[\begin{array}{lll}1.5 & 3 & 4.5\end{array}\right]$} \\
\hline Fast & $\mathrm{F}$ & {$\left[\begin{array}{lll}3 & 4.5 & 6\end{array}\right]$} \\
\hline Veryfast & VF & {$\left[\begin{array}{lll}4.5 & 6 & 7.5\end{array}\right]$} \\
\hline
\end{tabular}

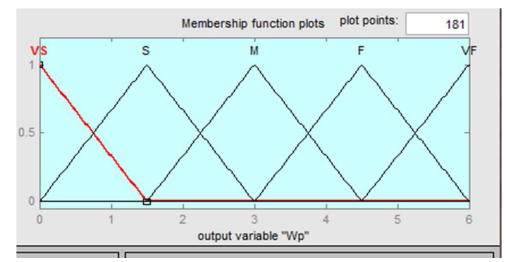

Hình 8. Miền xác định các hàm thành viên Distance.

\section{d. Xây dụng luật hợp thành}

Từ kinh nghiệm thực tế, các luật điều khiển của bánh trái và bảnh phải của Robot cho như sau:

Bảng 4. Bảng luật bánh phải.

\begin{tabular}{|c|c|c|c|c|c|}
\hline Angle & BN & N & Z & P & BP \\
\hline Distance & & & & & \\
\hline N & VS & S & VS & VF & VF \\
\hline M & VS & VS & S & VF & VF \\
\hline F & VS & S & F & VF & VF \\
\hline VF & VS & S & VF & VF & VF \\
\hline
\end{tabular}

Bảng 5. Bảng luật bánh trái.

\begin{tabular}{|c|c|c|c|c|c|}
\hline Angle & BN & N & Z & P & BP \\
\hline Z & VF & VF & VS & S & VS \\
\hline N & VF & VF & S & S & VS \\
\hline M & VF & VF & M & S & VS \\
\hline F & VF & VF & F & S & VS \\
\hline VF & VF & VF & VF & S & VS \\
\hline
\end{tabular}



trước.

Như vậy tổng cộng có tất cả 25 luật hợp thành để điều khiển robot cho bám quỹ đạo cho

\section{e. Chọn thiết bị hợp thành}

Đối với trường hợp bám quỹ đạo của robot di động, chọn theo phương pháp MAX - MIN.

\section{f. Chọn nguyên lí giải mò̀}

Giải mờ là quá trình xác định rõ giá trị đầu ra của bộ điều khiển. Việc chọn phương pháp giải mờ cũng ảnh hưởng đến đáp ứng hệ thống. Trong nghiên cứu này sử dụng phương pháp điểm trọng tâm (Centroid).

\section{KẾT QUẢ MÔ PHỎNG}

Để kiểm nghiệm thuật toán điều khiển, nghiên cứu chọn ra một số quỹ đạo điển hình bao gồm cả tuyến tính và phi tuyến. Chương trình mô phỏng được viết trên Matlab, simulink. Quỹ đạo gồm một số đoạn mà robot phải chuyển hướng chuyển động để đi tới đích. Kết quả mô phỏng cho giải thuật ứng dụng điều khiển mờ để bám quỹ đạo được chỉ ra trong Hình 9 (bám theo đường gấp khúc), trong đó màu xanh là quỹ đạo thiết kế, màu đỏ là quỹ đạo bám.

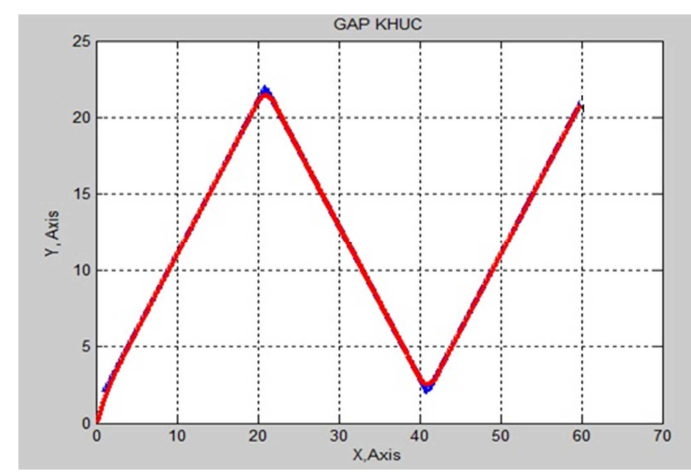

Hình 9. Xe chuyển động gấp khúc.

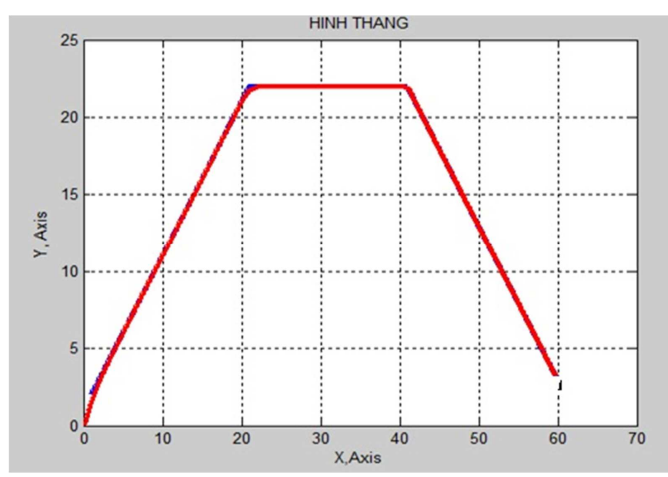

Hình 10. Chuyển động theo hình thang.

Robot bám theo các đường zich zắc có độ nghiêng các đoạn khác nhau với điểm xuất phát của robot là $(0,0)$. Từ kết quả nhận thấy ràng robot di chuyển khác ổn định theo quỹ đạo cho trước. Tại các đoạn đầu mút robot có chút sai lệch về vị trí do phải chuyển hướng đột ngột. Với các quỹ đạo hình thang, chũ̃ V (Hình 8), hình tròn (Hình 9), elip (Hình 10), ta cũng có kết quả với sai số rất nhỏ. Nhận thấy, điểm xuất phát của robot không ở trên quỹ đạo thiết kế, tuy nhiên bộ điều khiển đã tác động để robot chuyển động như mong muốn. 


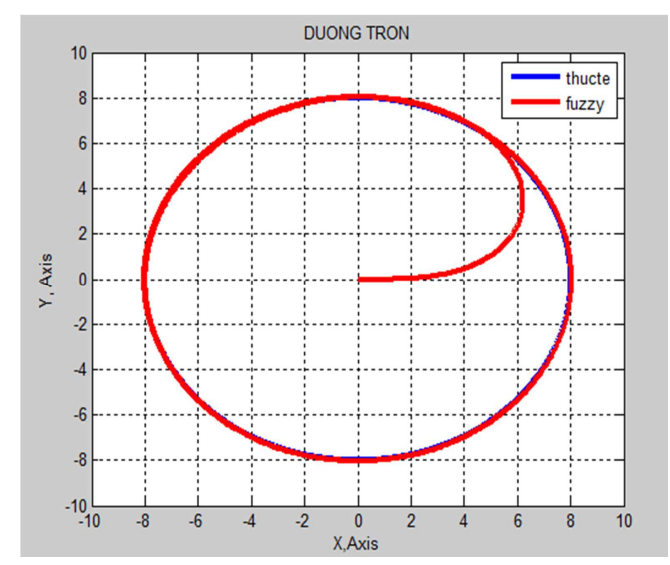

Hình 11. Chuyển động đường tròn $\mathrm{r}=8$.

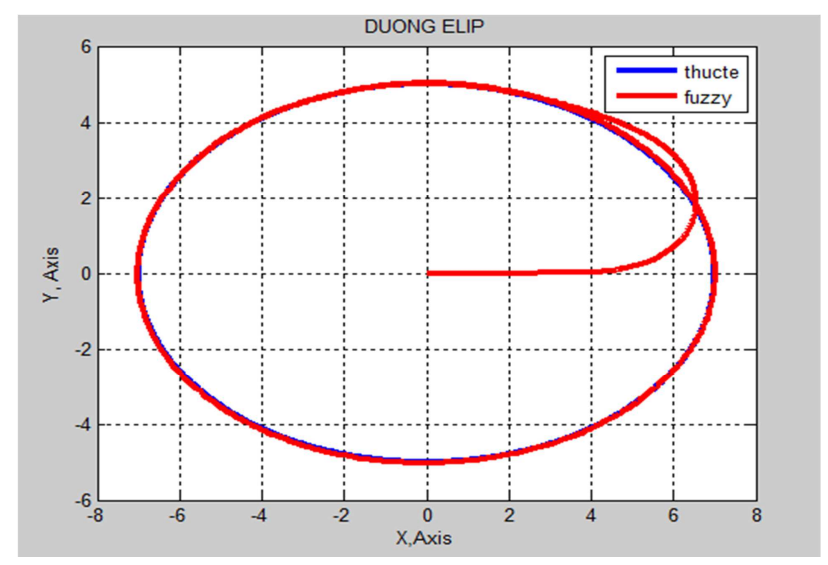

Hình 12. Chuyển động theo đường elip.

\section{KẾT LUẬN}

Nghiên cứu trình bày giải thuật điều khiển mờ để robot bám theo quỹ đạo cho trước. Các quỹ đạo tuyến tính, phi tuyến khác nhau được đưa ra để kiểm nghiệm tính đúng đắn của giải thuật. Kết quả chỉ ra robot di chuyển ổn định, sai số rất nhỏ trên các quỹ đạo tương đối phức tạp. Tác giả sẽ tiếp tục ở các hướng tiếp cận điều khiển robot thông minh khác như mạng neron, kết hợp mạng neron và logic mờ, đồng thời ứng dụng bộ điều khiển này cho robot thực.

\section{TÀI LIỆU THAM KHẢO}

1. Oubbati M., Schanz M., Levi P. - Kinematic and Dynamic Adaptive Control of a Nonholonomic Mobile Robot using a RNN, Proceedings 2005 IEEE International Symposium on Computatianal intelligence in Robotics and Automation, 2005, pp. 27-33.

2. Das T., Kar I. N., Chaudhury S. - Simple neuron- based adaptive controller for a nonholonomic mobile robot including actuator dynamics, Neurocomputing 69 (16-18) (2006) 2140-215.

3. Maalouf E., Saad M., and Saliah H. - A higher level path tracking controller for a fourwheel differentially steered mobile robot, Robotics and Autonomous Systems 54 (2006) 23-33.

4. Das T., Kar I. N. - Design and implementation of an adaptive fuzzy logic- based controller for wheeled mobile robots, IEEE Trans. Control Systems Technology 14 (3) (2006) 501-510.

5. Aguirre E. and Gonzales A. - Fuzzy beha viors for mobile robot navigation: design, coordination and fusion, Int. J. of Approximate Reasoning 25 (2000) 255-289.

6. Fatmi A., Yahmedi A. L., Khriji A. S., Masmoudi L. N. - A fuzzy logic based navigation of a mobile robot, World academy of science, Engineering and Technology 22 (2006) 169-174.

7. Fukazawa Y., Chomchana T., Ota J., Yuasa H., Arai T., and Asama H. - Region exploration pathplanning for a mobile robot expressing working environment by grid 
points, Proceedings of the 2003 IEEE International Conference on Robotics and Automation 2 (2003) 2448-2454.

8. Anmin Zhu and Simon X. Yang - A Fuzzy Logic Approach to Reactive Navigation of Behavior-based Mobile Robots, Proceedings of the 2004 IEEE/ASME International Conference on Robotics and Automation 5 (2004) 5045-5050.

9. Elnagar A. and Hussein A. M. - An adaptive motion prediction model for trajectory planner systems, Proceedings of the 2003 IEEE/ASME International Conference on Robotics and Automation 2 (2003) 2442-2447.

\title{
ABSTRACT
}

\section{PATH FOLLOWING OF MOBILE ROBOT USING FUZZY CONTROLLER}

\author{
Mac Thi Thoa*, Vu Van Thich, Tran Duc Trung \\ School of Mechanical Engineering, HUST, No. 1, Dai Co Viet, Hai Ba Trung, Hanoi
}

\section{Email: thoa.macthi@hust.edu.vn}

Fuzzy logic control, or intelligent control, demonstrates human ability in information processing. It is the origin of applying artificial technology in control area. This research presents fuzzy-based controller for an autonomous mobile robot navigation. First, the kinematic of mobile robot kinematic is developed, then modeling for simulation of tracking predetermined trajectory in robot workspace is performed. The simulation results illustrate the precision and efficiency of designed controller algorithm.

Keywords: path planning, fuzzy control, mobile robot, tracking trajectory, kinematic controller. 